\title{
The Nonsequential Fusion Method for Localization from Unscented Kalman Filter by Multistation Array Buoys
}

\author{
Gou Yanni and Wang Qi \\ College of Marine Science and Technology, Northwestern Polytechnical University, Xi'an 710072, China \\ Correspondence should be addressed to Gou Yanni; googogou@nwpu.edu.cn
}

Received 23 December 2015; Accepted 2 March 2016

Academic Editor: Filippo Cacace

Copyright ( 2016 G. Yanni and W. Qi. This is an open access article distributed under the Creative Commons Attribution License, which permits unrestricted use, distribution, and reproduction in any medium, provided the original work is properly cited.

\begin{abstract}
Based on special features of array buoy and the research field of location and tracking of underwater target, the research combines the highly adaptive nonlinear filtering algorithm unscented Kalman filter with the nonlinear programming of multistation array buoy positioning system. In accordance with the model of nonsequential target location, the research utilizes Unscented Transformation to update the measuring error and covariance matrix of state error, aiming at estimating the filtering of state variable and acquiring the object's current state of motion. The research analyzes the positioning performance of algorithm, pursuit path, astringency, and other performance indexes of target-relevant parameter through numerical simulation experiment. From the result, the conclusion that multistation array buoy can complete the task of tracing target track very well can be reached, which provides theoretical foundation for putting the algorithm into engineering practice.
\end{abstract}

\section{Introduction}

Sonobuoy is a very important part in integrated aviation antisubmarine, which is mainly used in large-scale exploratory search of enemy submarine. Sonobuoy is small in size and can explore large-area maritime space in short time. Sonobuoys have low requirements for the installation objects: they can be carried in quantity by airplanes and be equipped in helicopter, seaplane, or other fixed wing aircraft. Sonobuoy floats on the ocean and is slightly influenced by the noise of its carrier. Therefore, sonobuoy obtains the best detection effect.

One of buoy's major tasks is the effective location and tracking of underwater target [1]. Buoy's task is utilizing underwater sound sensors to conduct continuous measurement and estimation of targets' motion parameters such as orientation, speed, and course.

Traditional buoys mainly adopt liner array sensor to form wave beam in horizontal or vertical directions. Many buoys are arranged in the water to form geometric shape to explore underwater targets. The traditional method has low positioning accuracy and high requirements of relative location among buoys. The current randomness of buoys in the ocean current will influence the positioning accuracy and cause errors.
The new array buoy adopts plane array and can form wave beam in both horizontal and vertical directions, which improves the spatial gain and lower the working frequency. The combination of receiving array and buoy can greatly improve the noise detection of buoy system and obtain accurate measurement information. Theoretically, single array buoy can measure the location of target. However, because of the complexity and diversity of missions, the need of optimizing performance index of single array buoy cannot be satisfied. Therefore, researches begin to arrange multistation array buoys according to certain formations and integrate the data from multisensor nodes to locate the underwater targets accurately [2-6]. Compared with detecting result of single-station array buoy, multistation array buoy is equipped with acoustic aperture, which can obtain more reliable and accurate estimation information.

The centralized structure of fusion model combines the measurement data coming from different sensors into the fusion center and then the fusion center will process all measurement data together. Because of the huge amount of data, the centralized structure has high requirements for the processing ability of central nodes. However, compared with distributed and multisensor system, the centralized structure 


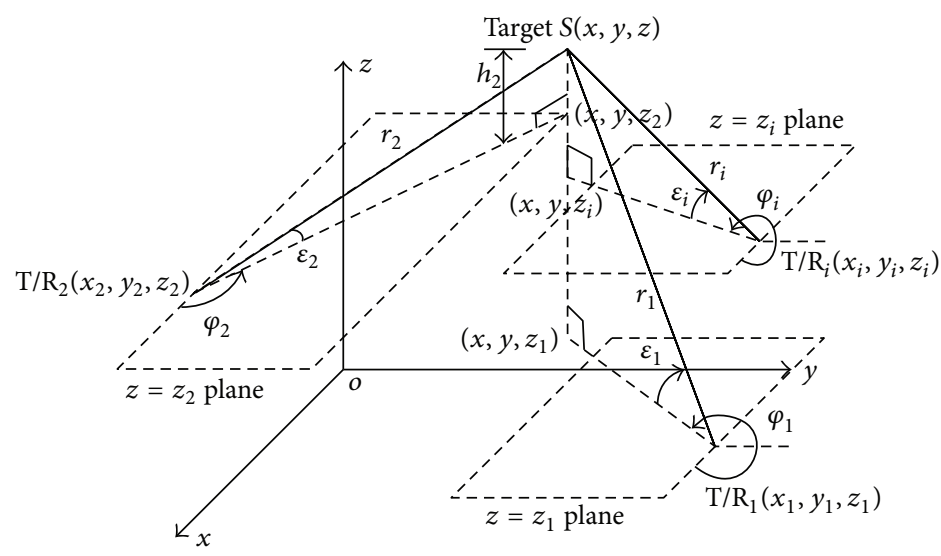

Figure 1: Multistation Array Geometry.

has higher accuracy thanks to the unified disposal of original data. With the rapid development of central processing unit, the target estimation and tracking issue which is based on centralized multisensor system, as a basic question of the information fusion among multisensor, has received more and more attention from researches.

In the centralized real-time processing and fusion model of time series information, buoys will transmit the observation data of target signals which comes from respective buoys to fusion processing center. However, in the complicate transmission process in maritime channel, out-of-sequence measurements (OOSM) problems will occur due to transport delay. For example, for the same object, the measurements of earlier time come later than the measurements of later time. Out-of-sequence measurement will negatively influence the reliability and accuracy of multiplatform fusion system. In order to solve the problem of OOSM, Bar-Shalom et al. $[7,8]$ put forward a series of suboptimal algorithms; the purpose is to use the out-of-sequence measurements for the current moment to update the target state, in order to obtain more accurate state estimation and its error covariance matrix. Zhou et al. [9] analyzed theoretically the optimality algorithm which is put forward by Bar-Shalom, point out that its optimality is associated with the discretization of process noise model, and propose an improved algorithm based on discrete time model, improving the accuracy of the filter. Bar-Shalom et al. [10] used the method of equivalent observation data in the original literature which extended one-step-lag OOSM to the multistep lag OOSM. The paper by Zhang et al. [11] was proposed based on the best linear unbiased estimation criterion of OOSM. Shen et al. [12] and Yu et al. [13] put forward solving the data fusion problem of multisensor OOSM based on one-step-lag OOSM.

In the real problem of tracking underwater target, Gaussian assumptions of the linear and noise of system model cannot be met. Therefore, the positioning and tracking issue of underwater target is a nonlinear system filter issue. Unscented Kalman filter (UKF) algorithm, based on Unscented Transformation (UT) and Kalman filter technology, selects some sampling points and approximates the mean value and variance through nonlinear variation. UKF not only makes nonlinear system filter reach high accuracy, but also adapts the noise very well. Therefore, UKF can be used to optimize the problem of OOSM filter fusion. When OOSM occurs, adopting OOSM algorithm which is based on UT to conduct current state estimation and update covariance will get a more accurate state estimation and its covariance. In order to improve the robustness during the operation of this algorithm, there appears square root unscented Kalman filtering algorithm. In order to add the nonlinear transformation of a noise distribution to a state estimation process, Wu et al. [14] give unscented Kalman filtering algorithm of a dimension expanding form. Simo $[15,16]$ deduces a smoothing algorithm of unscented Kalman filtering algorithm. Directing at interactive target moving model, Babu et al. [17] and Xu and Han [18] introduce UKF into the trace analysis of a maneuvering target by a passive sonar.

This paper is organized as follows. We discuss the localization principle and UKF filtering algorithm by multiarray buoys in Sections 2 and 3. Section 4 derived the nonsequential fusion UKF algorithm. In Section 5, simulation results are briefly described, and Section 6 is a conclusion.

\section{The Positioning Technology of Multistation Array Buoy}

2.1. Geometric Model. The site location and system equipment of multiarray buoys are shown as Figure 1. Currently, as for the operational command of sonarmen, the measurement of targets' three-dimensional coordinate is finished through the cooperation of two-dimensional sonar and heightmeasurement sonar. Two-dimensional sonar can measure the location and distance of targets. Height-measurement sonar can conduct supplementary measurement of height, which is accurate, simple, easy to control, and low in cost.

In Figure 1, the positioning system of $(\mathrm{T} / \mathrm{R})_{i}$ multistation array buoy consists of $i$ single-station array buoys. T represents the transmitting station and $\mathrm{R}$ represents the receiving station. $\mathrm{T} / \mathrm{R}_{i}$ is $\mathrm{T} / \mathrm{R}_{2}\left(x_{2}, y_{2}, z_{2}\right) \cdots \mathrm{T} / \mathrm{R}_{i}\left(x_{i}, y_{i}, z_{i}\right) S(x, y, z)$.

2.2. Positioning Principle. The stations of $(\mathrm{T} / \mathrm{R})_{i}$ multistation array buoy cooperate with each other; when measuring 


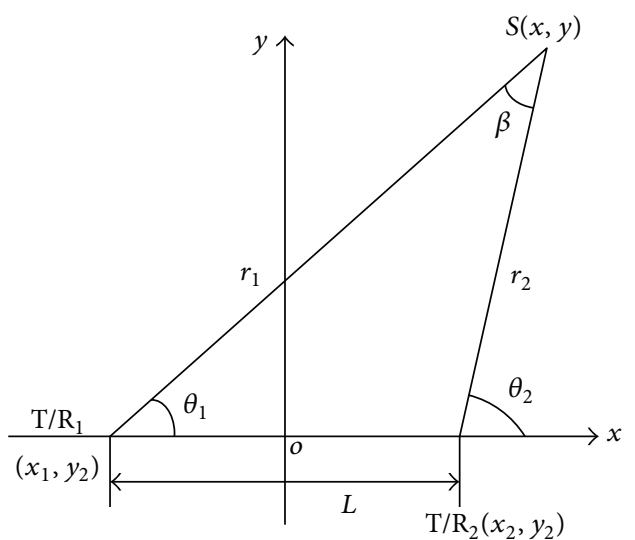

FIGURE 2: Double-station array buoy two-dimensional positioning principle.

the target's direction, azimuth angle information $\left(\theta_{1}, \theta_{2} \cdots \theta_{i}\right)$ could be simply used for positioning. See Figure 2 .

The system model takes the base line between two buoys as $x$-axis, lets the midpoint be the origin of coordinates, and establishes a rectangular coordinate system. The positioning equation is

$$
\begin{aligned}
& \theta_{1}=\tan ^{-1}\left[\frac{\left(y-y_{1}\right)}{\left(x-x_{1}\right)}\right], \\
& \theta_{2}=\tan ^{-1}\left[\frac{\left(y-y_{2}\right)}{\left(x-x_{2}\right)}\right] ;
\end{aligned}
$$

solving (1) to acquire the target location,

$$
\begin{aligned}
& x=\frac{\left(y_{1}-y_{2}\right)+x_{2} \tan \theta_{2}-x_{1} \tan \theta_{1}}{\tan \theta_{2}-\tan \theta_{1}}, \\
& y=y_{1}+\frac{\left(y_{1}-y_{2}\right)+\left(x_{2}-x_{1}\right) \tan \theta_{2}}{\tan \theta_{2}-\tan \theta_{1}} \tan \theta_{1} .
\end{aligned}
$$

2.3. Positioning Error Analysis. Under the assumed conditions, angle measuring errors of the two buoys and a station measuring error are $\sigma_{\theta_{1}}, \sigma_{\theta_{2}}$, and $\sigma_{s}$. To keep them constant, the positioning error equation (3) as shown below is differentiating from (2):

$$
\left[\begin{array}{l}
\mathrm{d} \theta_{1} \\
\mathrm{~d} \theta_{2}
\end{array}\right]=\left[\begin{array}{cc}
-\frac{\sin \theta_{1}}{r_{1}} & \frac{\cos \theta_{1}}{r_{1}} \\
-\frac{\sin \theta_{2}}{r_{2}} & \frac{\cos \theta_{2}}{r_{2}}
\end{array}\right]\left[\begin{array}{l}
\mathrm{d} x \\
\mathrm{~d} y
\end{array}\right]+\left[\begin{array}{l}
k_{\theta, 1} \\
k_{\theta, 2}
\end{array}\right],
$$

wherein

$$
\begin{aligned}
k_{\theta, 1} & =\frac{\sin \theta_{1}}{r_{1}} \mathrm{~d} x_{1}-\frac{\cos \theta_{1}}{r_{1}} \mathrm{~d} y_{1}, \\
k_{\theta, 2} & =\frac{\sin \theta_{2}}{r_{2}} \mathrm{~d} x_{2}-\frac{\cos \theta_{2}}{r_{2}} \mathrm{~d} y_{2}, \\
r_{\mathrm{i}} & =\sqrt{\left(x-x_{i}\right)^{2}+\left(y-y_{i}\right)^{2}} .
\end{aligned}
$$

Equation (3) is written as a matrix form:

$$
\mathrm{d} V=C \mathrm{~d} X+\mathrm{d} X_{s}
$$

wherein $\mathrm{d} V=\left[\begin{array}{ll}\mathrm{d} \theta_{1} & \mathrm{~d} \theta_{2}\end{array}\right]^{T}, \mathrm{~d} X=\left[\begin{array}{ll}\mathrm{d} x & \mathrm{~d} y\end{array}\right]^{T}$, and $\mathrm{d} X_{s}=$ $\left[\begin{array}{ll}k_{\theta, 1} & k_{\theta, 2}\end{array}\right]^{T}$,

$$
C=\left[\begin{array}{cc}
-\frac{\sin \theta_{1}}{r_{1}} & \frac{\cos \theta_{1}}{r_{1}} \\
-\frac{\sin \theta_{2}}{r_{2}} & \frac{\cos \theta_{2}}{r_{2}}
\end{array}\right]
$$

solving (5) to acquire the error vector of the target location:

$$
\mathrm{d} X=C^{-1}\left[\mathrm{~d} V-\mathrm{d} X_{s}\right] .
$$

The relationships between the target location error in the rectangular coordinate system $(\mathrm{d} x, \mathrm{~d} y)$, the angle measuring error $\left(\mathrm{d} \theta_{1}, \mathrm{~d} \theta_{2}\right)$, and the station measuring errors $\left(\mathrm{d} x_{1}, \mathrm{~d} y_{1}\right)$ and $\left(\mathrm{d} x_{2}, \mathrm{~d} y_{2}\right)$ can be seen from (7).

The covariance matrix of the positioning error mentioned above is

$$
\begin{aligned}
P_{\mathrm{d} X} & =E\left[\mathrm{~d} X \mathrm{~d} X^{T}\right]=C^{-1}\left\{E\left[\mathrm{~d} V \mathrm{~d} V^{T}\right]\right. \\
& \left.+E\left[\mathrm{~d} X_{s} \mathrm{~d} X_{s}^{T}\right]\right\} C^{-T}, \\
\sigma_{x}^{2} & =\frac{1}{|C|^{2}}\left[\frac{\cos ^{2} \theta_{2}\left(\sigma_{\theta 1}^{2}+\sigma_{s}^{2} / r_{1}^{2}\right)}{r_{2}^{2}}\right. \\
& \left.+\frac{\cos ^{2} \theta_{1}\left(\sigma_{\theta_{2}}^{2}+\sigma_{s}^{2} / r_{2}^{2}\right)}{r_{1}^{2}}\right], \\
\sigma_{y}^{2} & =\frac{1}{|C|^{2}}\left[\frac{\sin ^{2} \theta_{2}\left(\sigma_{\theta 1}^{2}+\sigma_{s}^{2} / r_{1}^{2}\right)}{r_{2}^{2}}\right. \\
& \left.+\frac{\sin ^{2} \theta_{1}\left(\sigma_{\theta_{2}}^{2}+\sigma_{s}^{2} / r_{2}^{2}\right)}{r_{1}^{2}}\right]
\end{aligned}
$$

wherein $|C|=\sin \left(\theta_{2}-\theta_{1}\right) / r_{1} r_{2}$.

2.4. The Geometric Interpretation of Positioning Precision. Any positioning system has different positioning precisions for targets in different spatial positions, which means the positioning error of a target location is closely related to the geometrical relationship of the target relative to a positioning station. Positioning stations with different geometric distributions have different positioning errors for targets in the same spatial positions. Therefore, the relationship between positioning errors and the geometric distributions of positioning stations has to be studied. Meanwhile, under that condition that the geometric distributions of positioning stations have been determined, knowing the positioning errors distributions of this positioning system for different spatial positions is necessary for effectively using the positioning system and accurately positioning and tracking a target.

In order to describe the relationship between a positioning error and geometry and measuring the influence of 
a geometric position on positioning performance, a noun, "geometric dilution of precision" (short for GDOP), is defined and can be expressed in a two-dimensional surface as follows:

$$
\mathrm{GDOP}=\left[\sigma_{x}^{2}+\sigma_{y}^{2}\right]^{1 / 2}
$$

Geometric dilution of precision describes the relationship between the positioning error and the base station geometric distribution participating in the positioning. It can become a standard studying the distribution regularities of positioning errors in different geometric distributions, and it can also become a reference for choosing the position of a base station when establishing a new buoy system.

\section{The Filtering Algorithm Based on UKF}

3.1. UT Transforming Principle. The traditional method [19] has low positioning accuracy and high requirements of relative location among buoys. The current randomness of buoys in the ocean current will influence the positioning accuracy and cause errors.

UKF is a filtering algorithm [20] combining UT (Unscented Transformation) and Kalman filtering technique. By specially selecting some Sigma sampling points and better approximating the mean and variance of random variables after nonlinear transformation, UKF can not only make the nonlinear system filter arrive at a higher precision, but also adapt well to a noise.

UKF filter firstly applies UT to a state equation and then estimates the filter by transformed state variables. UT is selecting some points in an original state distribution according to certain rules to make matrix and covariance of these points equal the mean and covariance of the original state distribution and to substitute these points into a nonlinear system function; thus acquire data points of a relevant nonlinear function; finally evaluate the transformed mean and covariance according to the point set.

Assume that there is an $n$-dimension random vector $x \sim$ $N(\bar{x}, P) ; m$-dimension random vector $z$ is one nonlinear function of $x, z=f(x)$; the statistical property of $x$ is $\left(\bar{x}, P_{x}\right)$; and the statistical property of $y,\left(\bar{z}, P_{z}\right)$ is transmitted by a nonlinear function $f(x)$.

The transformation is formulating a certain number of points $\xi_{i}(i=0,1, \ldots, L)$ based on amount of information $\left(\bar{x}, P_{x}\right)$, and these points are called points $\sigma$. Calculating the results of points $\sigma, \gamma_{i}(i=0,1, \ldots, L)$, transmitted by $f(x)$, and calculating $\left(\bar{z}, P_{z}\right)$ based on $\gamma_{i}(i=0,1, \ldots, L)$, commonly the number of points $\sigma$ is $2 n+1$; that is, $L=2 n$. The transformation is as the following two steps.

\subsubsection{Evaluating Points $\sigma$ and Their Weight. Consider}

$$
\begin{aligned}
& \xi_{0}=\bar{x} \\
& \xi_{i}= \begin{cases}\bar{x}+\left(\sqrt{(n+\lambda) P_{x}}\right)_{i}, & i=1,2, \ldots, n, \\
\bar{x}-\left(\sqrt{(n+\lambda) P_{x}}\right)_{i-n}, & i=n+1, n+2, \ldots, 2 n,\end{cases}
\end{aligned}
$$

$$
\begin{aligned}
& \omega_{0}^{m}=\frac{\lambda}{(n+\lambda)} \\
& \omega_{0}^{(c)}=\frac{\lambda}{(n+\lambda)}+\left(1-\alpha^{2}+\beta\right) \\
& \quad \omega_{i}^{m}=\omega_{i}^{(c)}=\frac{0.5}{(n+\lambda)}, i=1,2, \ldots, 2 n,
\end{aligned}
$$

wherein $\lambda=\alpha^{2}(n+\kappa)-n$; $n$ is the dimensionality of the state vector $x$; a scale parameter $\alpha$ determines the dispersion degree of points $\sigma$; and $\alpha$ is usually a small positive number. In order to ensure that the covariance matrix is a positively definite matrix, $\kappa$ is usually $0 . \beta$ describes the distribution information of state $x$ (if the distribution information of state $x$ follows Gaussian distribution, the optimal value of $\beta$ is $0)$. $\left(\sqrt{(n+\lambda) P_{x}}\right)$ represents the line of Cholesky resolution. $\omega_{i}^{(m)}(i=0,1, \ldots, 2 n)$ and $\omega_{i}^{(c)}(i=0,1, \ldots, 2 n)$ represent the weights of a first-order statistical property and a second-order statistical property, respectively.

3.1.2. Calculating the Results of Points $\sigma, \gamma_{i}(i=0,1, \ldots, L)$, Transmitted by $f(x)$, and Acquiring (11). Consider

$$
\begin{aligned}
\gamma_{i} & =f\left(\xi_{i}\right), \quad i=0,1, \ldots, 2 n, \\
\bar{z} & =\sum_{i=0}^{2 n} \omega_{i}^{(m)} \gamma_{i}, \\
P_{z} & =\sum_{i=0}^{2 n} \omega_{i}^{(c)}\left(\gamma_{i}-\bar{z}\right)\left(\gamma_{i}-\bar{z}\right)^{T}, \\
P_{x z} & =\sum_{i=0}^{2 n} \omega_{i}^{(c)}\left(\zeta_{i}-\bar{x}\right)\left(\gamma_{i}-\bar{z}\right)^{T} .
\end{aligned}
$$

UT is different from Monte Carlo method. The former is selecting a few points $\sigma$ from the given distribution based on the certain rules but not determining the points by random sampling. In addition, the UT is not a common method of weighting, so it is also not a sampling statistics.

3.2. UKF Filtering Algorithm. If using UT to replace the local linearization means in EKF algorithm, UKF filtering algorithm can be acquired. The equation of UKF filter is as follows:

(A) For a given state $\widehat{x}_{k-1 \mid k-1}$ and a covariance matrix $P_{k-1 \mid k-1}$, it is easy to acquire a one-step predicted value $\hat{x}_{k \mid k-1}$ and a covariance matrix $P_{k \mid k-1}$ for forecasting an error based on a target movement model:

$$
\begin{aligned}
& \hat{x}_{k \mid k-1}=\Phi_{k-1} \cdot \hat{x}_{k-1 \mid k-1}, \\
& P_{k \mid k-1}=\Phi_{k-1} \cdot P_{k-1 \mid k-1} \cdot \Phi_{k-1}^{T}+G_{k-1} \cdot Q_{k-1} \cdot G_{k-1}^{T} .
\end{aligned}
$$

(B) Evaluating information $\hat{x}_{k \mid k-1}$ and $P_{k \mid k-1}$ of points $\sigma$ by applying UT, calculating $\widehat{x}_{k \mid k-1}$ and $P_{k \mid k-1}$ of 
points $\sigma$ by the transmission result of a nonlinear measurement equation, and having the following equations through the transmission of $x_{k}$ by the measurement equation,

$$
\begin{array}{rl}
\xi_{k}^{(0)} & =\widehat{x}_{k \mid k-1}, \\
\xi_{k}^{(i)} & =\widehat{x}_{k \mid k-1}+\left(\sqrt{(n+\lambda) P_{k \mid k-1}}\right)_{i} \quad i=1,2, \ldots, n, \\
\xi_{k}^{(i)}=\widehat{x}_{k \mid k-1}+\left(\sqrt{(n+\lambda) P_{k \mid k-1}}\right)_{i-n} & \\
i & i=n+1, n+2, \ldots, 2 n .
\end{array}
$$

Calculating a measurement one-step prediction, a covariance of a measurement error, and a crosscovariance matrix between a state error and a measurement error after UT, that is,

$$
\begin{aligned}
z_{k}^{(i)} & =h_{k}\left(\zeta_{k}^{(i)}\right), \\
\widehat{z}_{k \mid k-1} & =\sum_{i=0}^{2 n} \omega_{i}^{(m)} z_{k}^{(i)}, \\
P_{z z} & =\sum_{i=0}^{2 n} \omega_{i}^{(c)}\left(z_{k}^{(i)}-\widehat{z}_{k \mid k-1}\right)\left(z_{k}^{(i)}-\widehat{z}_{k \mid k-1}\right)^{T}, \\
P_{x z} & =\sum_{i=0}^{2 n} \omega_{i}^{(c)}\left(\zeta_{k}^{(i)}-\widehat{x}_{k \mid k-1}\right)\left(z_{k}^{(i)}-\widehat{z}_{k \mid k-1}\right)^{T} .
\end{aligned}
$$

(C) After acquiring a new measurement value $z_{k}$, updating the filter, and acquiring the update value of the filter and the covariance matrix of an evaluated error,

$$
\begin{aligned}
\hat{x}_{k \mid k} & =\widehat{x}_{k \mid k-1}+W_{k}\left(z_{k}-\widehat{z}_{k \mid k-1}\right), \\
W_{k} & =P_{x z} P_{z z}^{-1}, \\
P_{k \mid k} & =P_{k \mid k-1}-W_{k} P_{z z} W_{k}^{T},
\end{aligned}
$$

wherein $W_{k}$ is a gain matrix of the filter.

\section{Nonsequential Fusion UKF Algorithm}

4.1. Fusion Model. A centralized fusion structure sends measurement values given by all the not fixed coding sensors after observing targets to a fusion center, and let a center processor uniformly process this measurement information. In the information fusion center, the observation platforms of sensors are numbered consecutively combined with a sequential fusion idea.

First, predict and update the state in the first sensor. To be clear that the first sensor is not a single sensor but refers to the first to be processing nodes and is not necessarily the same sensor in every processing time. Then input the updated result of the first sensor as the updated state of the second sensor and continue updating. Similarly, update the values of state estimation using the measurement values of many

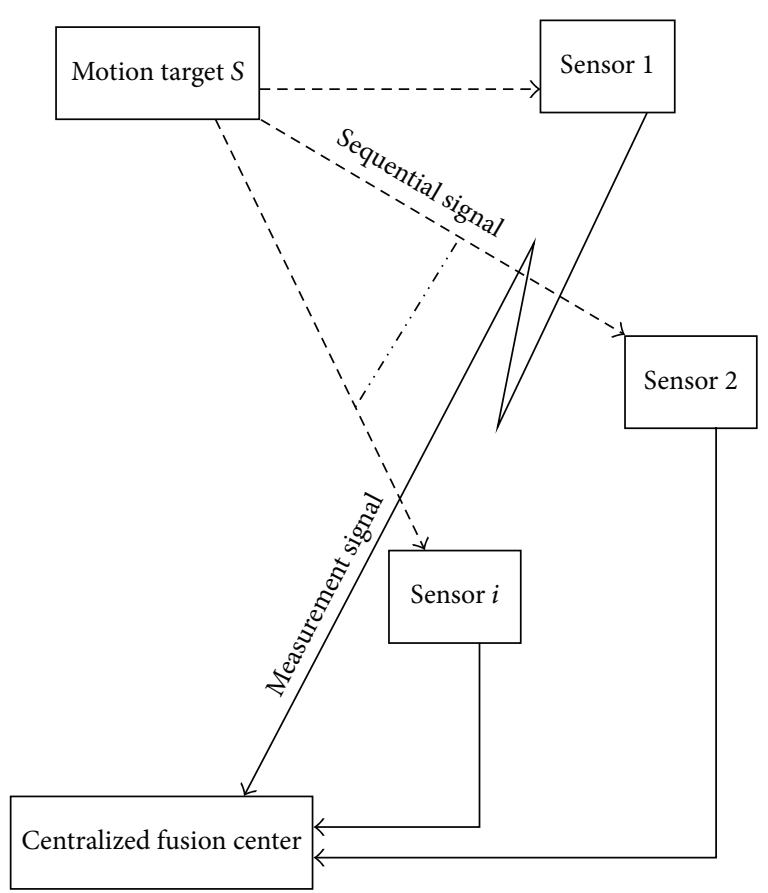

FIGURE 3: Diagram illustrating centralized structure.

sensors and ensure that the updated sequence of the sensors would not affect the final result of fusion during the process of updating. The purpose is to ensure the precision of estimated results based on certain calculation complexity. As for out-ofsequence centralized fusion structure, see Figure 3.

4.2. Out-of-Sequence UKF Algorithm. The state equation and measurement equation of underwater multistation buoy array of nonlinear system model can be expressed as follows:

$$
\begin{aligned}
x_{k+1} & =f\left(x_{k}, q_{k}\right), \\
y_{k} & =h\left(x_{k}\right)+r_{k},
\end{aligned}
$$

wherein $f$ is the state transition function, $h$ is the measurement function, $q_{k} \sim N\left(0, Q_{k}\right)$ is the process noise, and $r_{k} \sim$ $N\left(0, R_{k}\right)$ is measurement noise and also is the innovation. Those two noises, irrelevant to each other, are zero mean gauss noises.

When using UKF algorithm, first we need to define the extended state and the covariance matrix as

$$
\begin{aligned}
& \hat{x}_{k}^{a}=\left[\begin{array}{c}
\widehat{x}_{k} \\
\widehat{q}_{k} \\
\widehat{r}_{k}
\end{array}\right], \\
& P_{k}^{a}=\left[\begin{array}{ccc}
P_{k} & 0 & 0 \\
0 & Q_{k} & 0 \\
0 & 0 & R_{k}
\end{array}\right]
\end{aligned}
$$

according to formula (10) to get $2 n_{a}+1$ sampling points $\zeta_{i, k}^{x}$ and the weight allocation of sampling points $\omega_{i}^{(m)}$. 
By using UT conversion, information prediction equation can be acquired as formula (14). Defined $i_{k}$ is information state contribution and $I_{k}$ is the covariance matrix of $i_{k}$ as

$$
\begin{aligned}
i_{k} & =H_{k}^{T} R_{k}^{-1}\left[r_{k}+H_{k} \widehat{x}_{k \mid k-1}\right], \\
I_{k} & =H_{k}^{T} R_{k}^{-1} H_{k},
\end{aligned}
$$

wherein $H_{k}$ is the Jacobian matrix, $Q_{k}$ is process noise covariance, and $R_{k}$ is measurement noise covariance and $R_{k}=$ $y_{k}-h\left(\widehat{x}_{k \mid k-1}\right)$.

Therefore information contribution value can be updated as

$$
\begin{aligned}
& i_{k+1}=H_{k+1}^{T} R_{k+1}^{-1}\left[r_{k+1}+H_{k+1} \widehat{x}_{k+1 \mid k}\right] \\
& I_{k+1}=H_{k+1}^{T} R_{k+1}^{-1} H_{k+1} .
\end{aligned}
$$

On the condition of multistation buoy observation, the information contribution value of No. $s(s=1,2, \ldots, s)$ buoy is

$$
\begin{aligned}
& i_{k+1}^{s}=H_{k+1}^{s}\left(R_{k+1}^{-1}\right)^{-1}\left[r_{k+1}^{s}+H_{k+1} \widehat{x}_{k+1 \mid k}\right], \\
& I_{k+1}^{s}=\left(H_{k+1}^{s}\right)^{T}\left(R_{k+1}^{-1}\right)^{-1} H_{k+1}^{s} .
\end{aligned}
$$

Therefore, we can acquire the integrated result of multistation array buoy as follows:

$$
\widehat{z}_{k+1}=\widehat{z}_{k+1 \mid k}+\sum_{s=1}^{S} i_{k+1}^{s}
$$

\section{Numerical Simulation and Result Analysis}

According to the principle that double-station array buoy uses angle information to conduct two-dimensional positioning described in Section 3.1, we carried out simulation to positioning performance of algorithm. The parameters when making simulation are the following: the target moves towards $x$ direction $\pm 20 \mathrm{~km}$ and towards $y$ direction $\pm 20 \mathrm{~km}$, underwater sound velocity $v_{c}=1.5 \mathrm{~km} / \mathrm{s}$, the coordinate of T/R station is $(-7.5,0) \mathrm{km}$, and $R$ station is $(7.5,0) \mathrm{km}$, angulation errors $\sigma_{\theta_{T}}=1.5 \mathrm{mrad}, \sigma_{\theta_{R}}=1.5 \mathrm{mrad}$, stations measuring error $\sigma_{s}=15 \mathrm{~m}$, Monte-Carlo: 500 times. We can get two-dimensional positioning error graph and threedimensional GDOP graph, as shown in Figures 4 and 5, respectively.

From the two abovementioned graphs, we can see that when positioning using double-station array buoy, the distribution of positioning error is as follows: the whole graph is a regular butterfly in shape, comprising three areas: baseline area (two array buoy connection parts), wide board area (the extended part outside the baseline), and side board area (the extended part with two stations as vertex). The positioning precision in the baseline area is worse; the wide board area has the typical feature of bistatic sonar system with balanced error distribution; GDOP isoline extends outside from the baseline with slowly decreased precision, covering most of areas; side board area is the worst area in positioning precision.

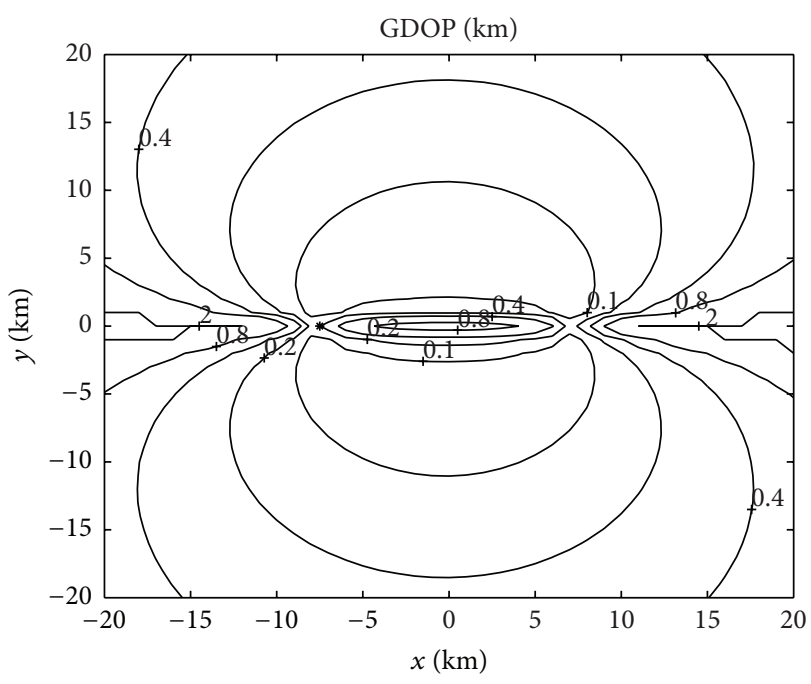

FIGURE 4: Spatial distribution of double-station array buoy twodimensional target positioning error.

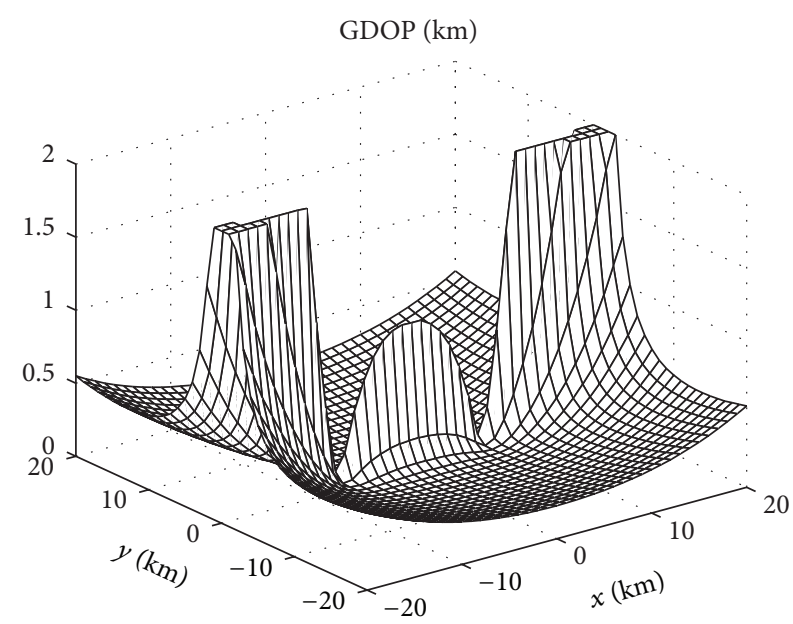

FIGURE 5: Distribution graph of three-dimensional space.

Based on the analysis on abovementioned positioning and UKF filtering step described in Section 3.2, we conducted numerical simulation to the tracking effect of double-station array buoy. The parameters of simulation are as follows: acoustic velocity: $1500 \mathrm{~m} / \mathrm{s}$, sampling interval: $1 \mathrm{~s}$, simulation length sampling for 1000 times, buoy direction-finding error of mean square deviation: $5 \pi / 180$, frequency measurement error of mean square deviation: $0.1 \mathrm{~Hz}$, target initial position: (-2500, 4500), velocity: $10 \mathrm{~m} / \mathrm{s}$, course: $3 \pi / 4$, and frequency: $1000 \mathrm{~Hz}$, and the mean square deviations of the disturbance noises from velocity, course, and frequency are all defined as 0.1. Figures 6 and 8 show the simulation results of the simulation matrix of determinant, in which the doublestation and four-station array buoys are vertical to the target course. Figures 7 and 9 show the estimation astringency curves of relevant parameters including velocity, frequency, and course. 


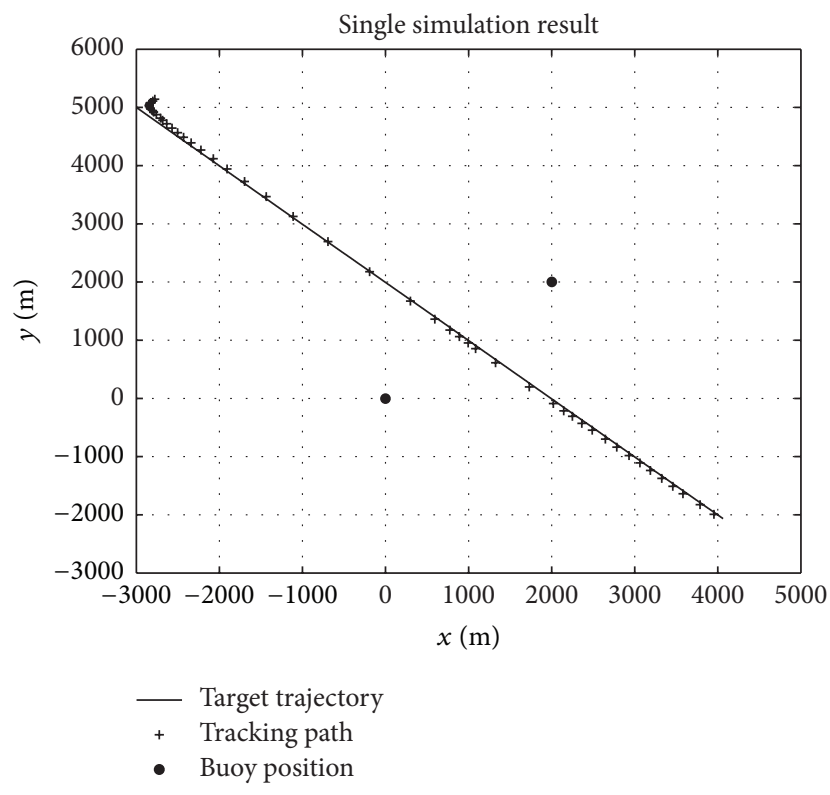

FIGURE 6: Schematic diagram of a single simulation tracking.
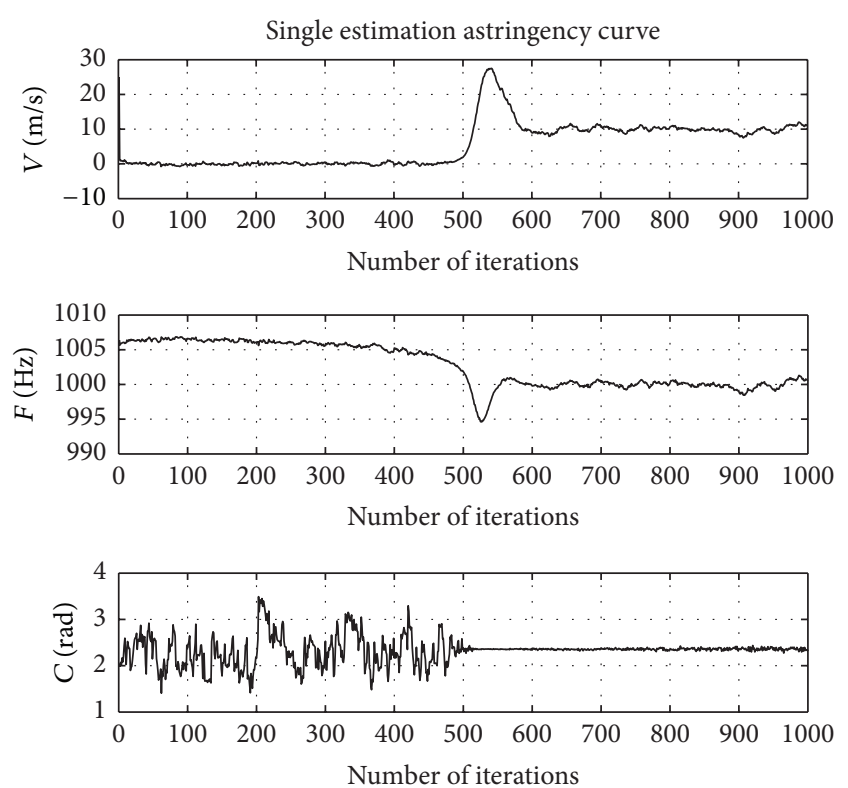

FIGURE 7: Estimation astringency curve of speed, frequency, and course.

In order to obtain the data about the changes of target frequency over time, we use short-time Fourier transform method to estimate frequency. For each FFT, transform the lengths of 256 sampling points with $50 \%$ overlapping ratio and 1024 algorithm length.

After obtaining FFT of each interval, select the largest frequency point as the measured frequency of the interval. Conduct smooth filtering to the calculated frequency signal using 32-pecking-order-number of FIR low-pass filter with $100 \mathrm{~Hz}$ low pass cut-off frequency.

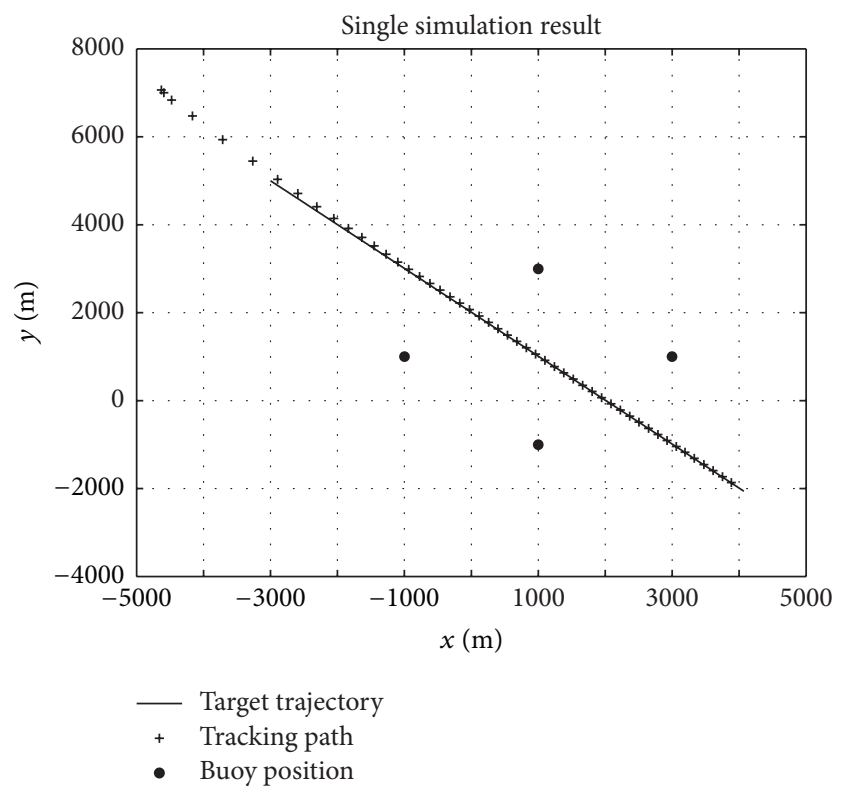

FIGURE 8: Schematic diagram of a single simulation tracking.
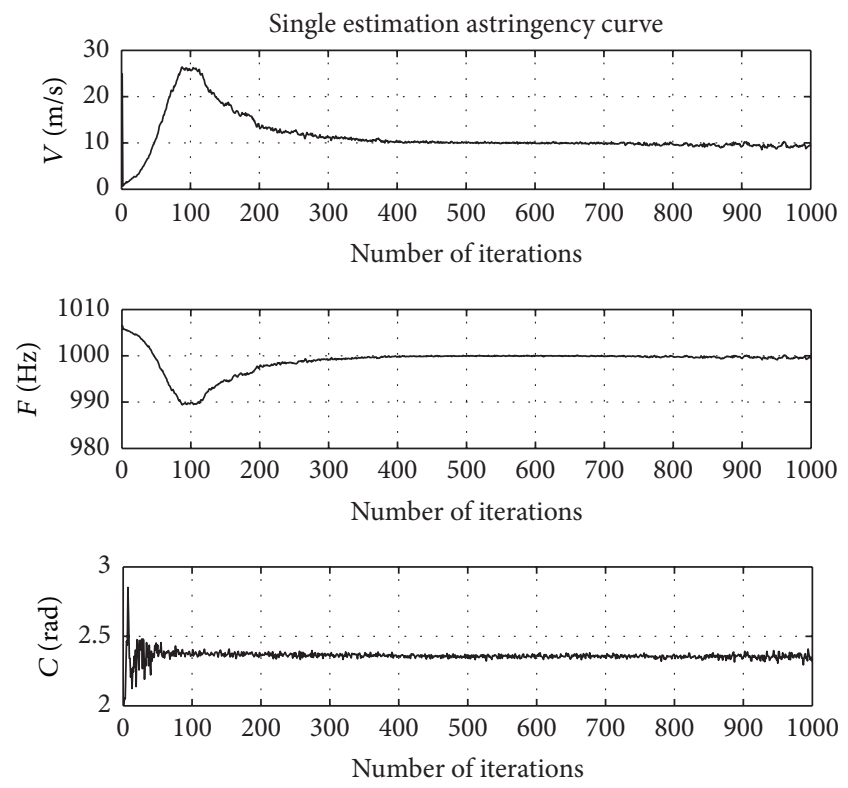

FIGURE 9: Estimation astringency curve of speed, frequency, and course.

From Figures 6 and 9, we can see that the proposed algorithm in this paper can track the target trajectory well and parameters such as target speed, frequency, and course can be converged efficiently. Contrasting Figures 7 and 9, the number of iterations decreased from 500 to 200 . The results show that multistation array buoy can effectively improve the computation efficiency.

In industry application, the cost of dropping buoy is one of the factors we must take into consideration. Therefore, it is reasonable to balance the accuracy requirements and computation time is very necessary. 


\section{Conclusion}

This paper studies the positioning and tracking of underwater moving target utilizing double-station array buoy and analyzed relevant performance under the framework of unscented Kalman filter (UKF) theory. The result shows that reasonable numerical simulation method can finish the task of tracking the target trajectory under the condition of simulation very well and the relevant parameters such as speed, course, and frequency have astringency. The conclusion of this paper aims to provide theoretical foundation for putting the algorithm into engineering practice.

In the process of target tracking, some false alarm and dismissal probability from measuring data may occur because of the influence of complex ocean environment. In this situation, the OOSM problem will become more complex, which requires more future researches on the nonlinear outof-sequence filtering problem.

\section{Competing Interests}

The authors declare that there is no conflict of interests.

\section{Acknowledgments}

This work was supported by the Young Scientists Fund of the National Natural Science Foundation of China (Grant no. 5130919).

\section{References}

[1] G. M. Ling and Z. M. Wang, "Sonar buoy technology and its development direction," Acoustic and Electronic Engineering, vol. 3, no. 1, pp. 1-5, 2007.

[2] O. Erdinc, P. Willett, and S. Corraluppi, "Multistatic sensor placement: a tracking approach," Journal of Advances in Information Fusion, vol. 2, no. 1, pp. 22-33, 2007.

[3] S. Coraluppi, "Multistatic sonar localization," IEEE Journal of Oceanic Engineering, vol. 31, no. 4, pp. 964-974, 2006.

[4] D. Grimmett and C. Wakayama, "Multistatic tracking for continous active sonar using Doppler-bearing measurements," in Proceedings of the 16th International Conference on Information Fusion (FUSION '13), pp. 258-265, Istanbul, Turkey, July 2013.

[5] K. X. Zhao, J. L. Liang, J. Karlsson, and J. Li, "Enhanced multistatic active sonar signal processing," in Proceedings of the 38th IEEE International Conference on Acoustics, Speech, and Signal Processing (ICASSP '13), pp. 3861-3865, Vancouver, Canada, May 2013.

[6] J. Ling, L. Xu, and J. Li, "Adaptive range-doppler imaging and target parameter estimation in multistatic active sonar systems," IEEE Journal of Oceanic Engineering, vol. 39, no. 2, pp. 290-302, 2014.

[7] Y. Bar-Shalom, M. Mallick, H. M. Chen, and R. Washburn, "One-step solution for the general out-of-sequencemeasurement problem in tracking," in Proceedings of the IEEE Aerospace Conference, vol. 4, pp. 1551-1559, Montana, Mont, USA, March 2002.

[8] Y. Bar-Shalom, "Update with out-of-sequence measurements in tracking: exact solution," IEEE Transactions on Aerospace and Electronic Systems, vol. 38, no. 3, pp. 769-778, 2002.
[9] W. H. Zhou, L. Li, G. H. Chen, and A. Yu, "Optimality analysis of one-step OOSM filtering algorithms in target tracking," Science in China, Series F: Information Sciences, vol. 50, no. 2, pp. 170187, 2007.

[10] Y. Bar-Shalom, H. M. Chen, and M. Mallick, “One-step solution for the multistep out-of-sequence-measurement problem in tracking," IEEE Transactions on Aerospace and Electronic Systems, vol. 40, no. 1, pp. 27-37, 2004.

[11] K. S. Zhang, X. R. Li, and Y. M. Zhu, "Optimal update with out-of-sequence measurements," IEEE Transactions on Signal Processing, vol. 53, no. 6, pp. 1992-2004, 2005.

[12] X. J. Shen, Y. M. Zhu, E. B. Song, and Y. Luo, "Optimal centralized update with multiple local out-of-sequence measurements," IEEE Transactions on Signal Processing, vol. 57, no. 4, pp. 1551-1562, 2009.

[13] A. X. Yu, D. N. Lian, W. D. Hu, and D. Zhen, "A unified outof-sequence measurements filter," in Proceedings of the IEEE International Radar Conference (RADAR '05), pp. 453-458, Arlington, Va, USA, May 2005.

[14] Y. X. Wu, D. W. Hu, M. P. Wu, and X. P. Hu, "Unscented Kalman filtering for additive noise case: augmented versus nonaugmented," IEEE Signal Processing Letters, vol. 12, no. 5, pp. 357-360, 2005.

[15] S. SÄrkkÄ, "Unscented Rauch-Tung-Striebel smoother," IEEE Transactions on Automatic Control, vol. 53, no. 3, pp. 845-849, 2008.

[16] S. Särkkä, "Continuous-time and continuous-discrete-time unscented Rauch-Tung-Striebel smoothers," Signal Processing, vol. 90, no. 1, pp. 225-235, 2010.

[17] V. S. Babu, M. R. Shankar, S. Majumdar, and S. K. Rao, "IMMunscented Kalman filter based tracking of maneuvering targets using active sonar measurements," in Proceedings of the International Conference on Communications and Signal Processing (ICCSP '11), pp. 126-130, Calicut, India, February 2011.

[18] Z. P. Xu and S. P. Han, "Application of UKF for passive tracking of maneuvering target based on bearings of two sonar sensors," in Proceedings of the 4th International Congress on Image and Signal Processing (CISP '11), pp. 2566-2570, Shanghai, China, October 2011.

[19] Z. X. Hu, M. T. Sun, and W. G. Su, "Simulation analysis of LOFIX fixing accuracy for passive omni-directional sonobuoy," Electronics Optics \& Control, vol. 16, no. 12, pp. 26-29, 2009.

[20] S. J. Julier and J. K. Uhlmann, "Unscented filtering and nonlinear estimation," Proceedings of the IEEE, vol. 92, no. 3, pp. 401422, 2004. 


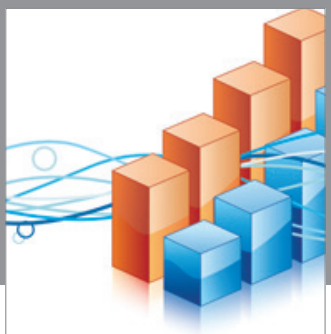

Advances in

Operations Research

vatem alat4

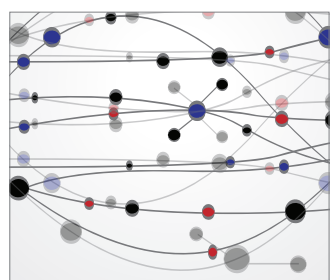

\section{The Scientific} World Journal
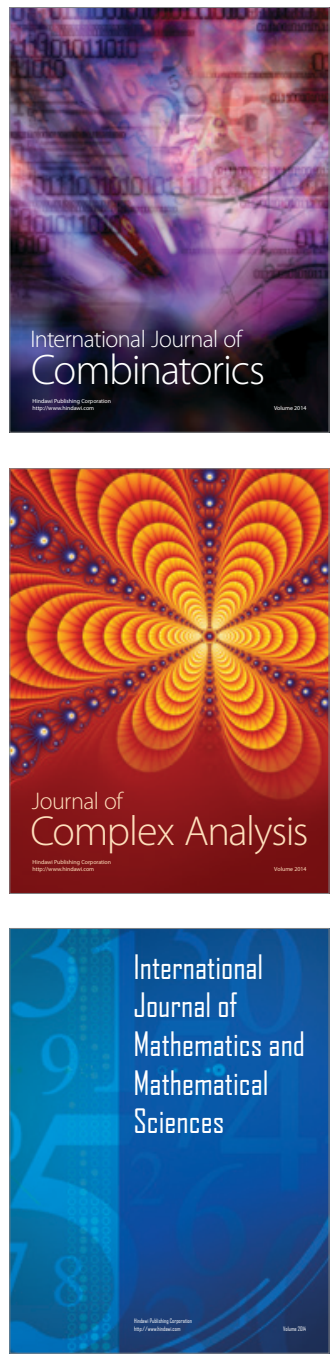
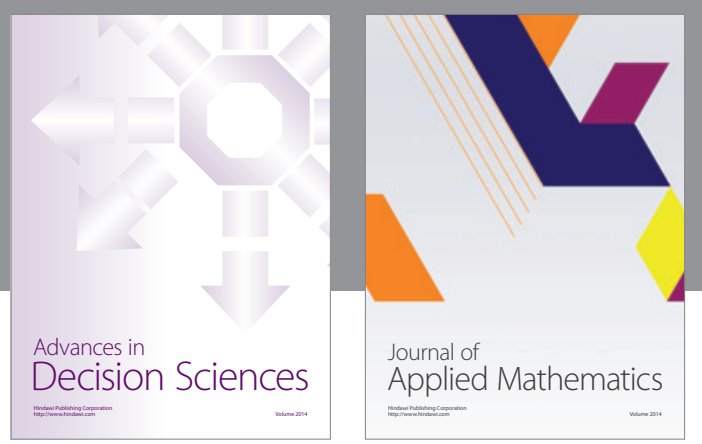

Algebra

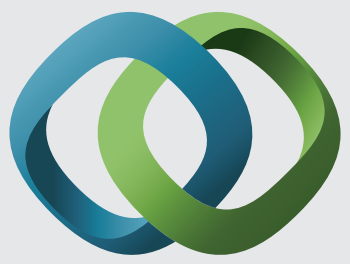

\section{Hindawi}

Submit your manuscripts at

http://www.hindawi.com
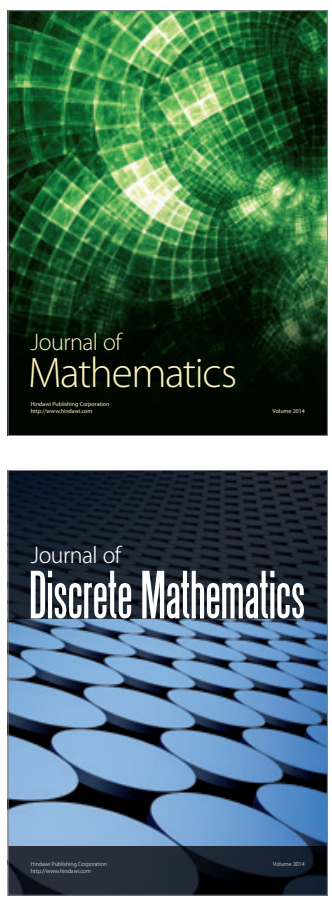

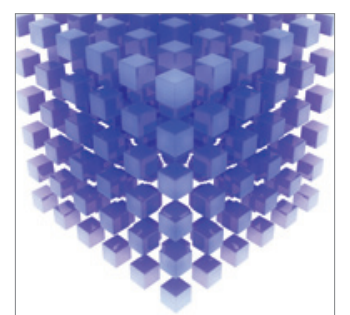

Mathematical Problems in Engineering
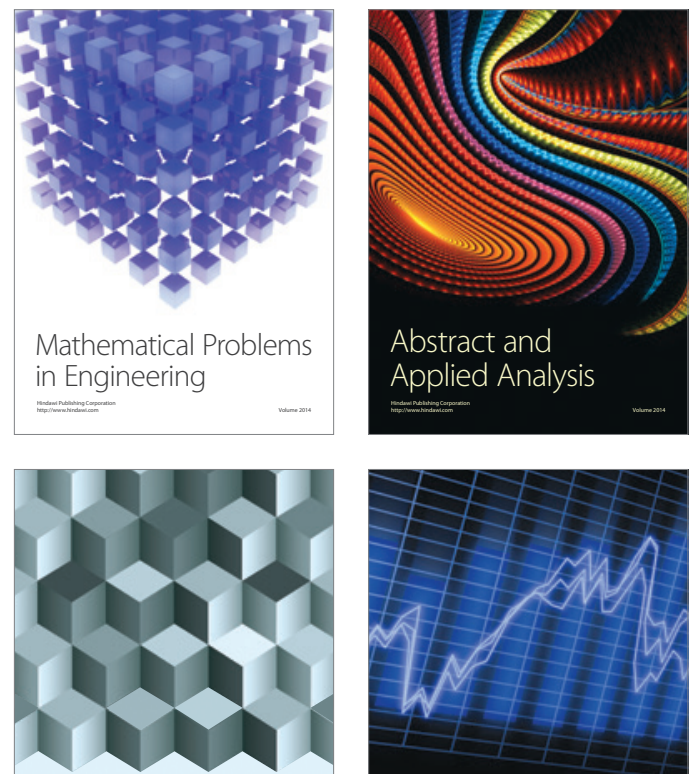

Journal of

Function Spaces

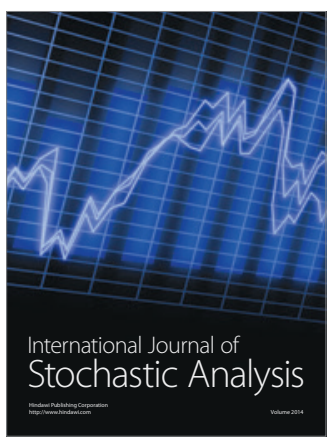

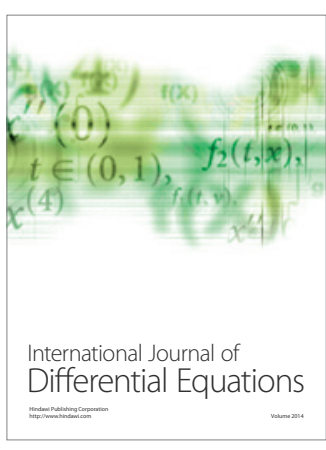
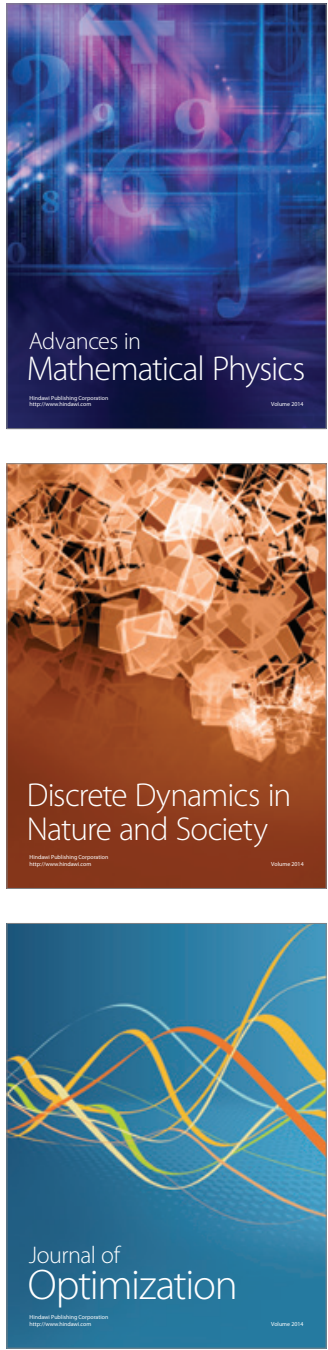